\title{
Contribution of Ni KLL Auger Electrons to the Probing Depth of the Conversion Electron Yield Measurements
}

\author{
Shinjiro Hayakawa, ${ }^{\dagger}$ Aya TanaKa, and Takeshi HirokaWa \\ Department of Applied Chemistry, Graduate School of Engineering, Hiroshima University, \\ 1-4-1 Kagamiyama, Higashi-Hiroshima, Hiroshima 739-8527, Japan
}

\begin{abstract}
The averaged attenuation length of emitted electrons with the conversion electron yield (CEY) method was evaluated for $\mathrm{Ni}$ films with the X-rays below and above the Ni K absorption edge. The evaluated attenuation length was $13.1 \mathrm{~nm}$ with the X-rays below the Ni K absorption edge, and it became $24.0 \mathrm{~nm}$ with the contribution of Ni KLL Auger electrons that had the attenuation length of $78.1 \mathrm{~nm}$. The probing depth of the CEY measurements are determined both the penetration depth of x-rays and the attenuation length of emitted electrons, and the modification of the probing depth was investigated with the grazing incidence condition. The glancing angle dependence of the CEY was compared with the model calculation, and the probing depth of around $1.6 \mathrm{~nm}$ was realized under the total reflection condition. The probing depth was controlled from $1.6 \mathrm{~nm}$ to the attenuation length by changing the glancing angle.
\end{abstract}

(Received September 5, 2009; Accepted December 11, 2009; Published February 10, 2010)

\section{Introduction}

X-ray absorption fine structure (XAFS) measurements are a unique analytical method that can provide the chemical state and local structure of the element of interest. Beside the conventional transmission method, X-ray fluorescence yield (XFY) or electron yield (EY) method is often employed to obtain XAFS spectra. Both XFY and EY originate from the decays of a core hole, and those methods can provide equivalent information to that obtained with the conventional transmission method. The EY detection is useful for XAFS measurements especially in the soft $\mathrm{x}$-ray region because the conventional transmission method is not practical, owing to the strong absorption cross section of a sample. ${ }^{1}$ Among various EY detection techniques, total electron yield (TEY) is the simplest method that collects emitted electrons of all kinetic energies from a sample. TEY is usually measured as an X-ray induced sample current that runs between the sample and the ground, and the measurements are carried out in the vacuum environment. $^{2-5}$ The EY can be measured under the gaseous environment, and the ionization of surrounding gas molecules by the emitted energetic electrons can be detected as conversion electron yield (CEY) with the use of an appropriate biased electrode. The role of the electrode is similar to those used in the gas counters for x-ray detection, ${ }^{6}$ and the mode of the operation is similar to the ionization chamber ${ }^{7-10}$ or to the proportional counter. ${ }^{11,12}$ Both TEY and CEY methods have surface or near surface sensitivity, and the probing depth of these methods is related to the inelastic mean free path (IMFP) ${ }^{13}$ of electrons. Kawai et al. have reported the probing depth of less than $1 \mathrm{~nm}$ with TEY method under the total reflection condition, and the probing depth was larger than $1 \mathrm{~nm}$ with the

† To whom correspondence should be addressed.

E-mail: hayakawa@hiroshima-u.ac.jp
Si KLL Auger electron yield in the same condition. ${ }^{3}$ It is known that the contribution of energetic Auger electrons makes the probing depth with the CEY method deeper, but the number of evaluated probing depths was relatively small. This paper reports the contribution of $\mathrm{Ni}$ KLL Auger electrons to the probing depth of the CEY detection; some ways of changing probing depth are also discussed.

\section{Theoretical}

The averaged attenuation length with TEY is defined as an averaged inelastic mean free path (IMFP), $\lambda$ of emitted electrons for a given kinetic energy and material.

$$
\tau_{\mathrm{TEY}}=\frac{\int N\left(E_{\mathrm{k}}\right) \lambda\left(E_{\mathrm{k}}\right) \mathrm{d} E_{\mathrm{k}}}{\int N\left(E_{\mathrm{k}}\right) \mathrm{d} E_{\mathrm{k}}}
$$

$N\left(E_{\mathrm{k}}\right)$ is the number of emitted electrons as a function of the kinetic energy $E_{\mathrm{k}}$.

CEY is measured with the gaseous environment, and the difference between the TEY and the CEY can be expressed with the gas amplification factor $G\left(E_{\mathrm{k}}\right)$. The gas amplification factor can be defined as a number of electrons created by a primary emitted electron of kinetic energy $E_{\mathrm{k}}$, and an appropriate electrode is utilized in the similar manner with an ionization chamber to prevent created electron-ion pairs from recombination. The typical ionization energies of gas molecules are 41.3 and $34.8 \mathrm{eV}$ per electron-ion pair for $\mathrm{He}$ and $\mathrm{N}_{2}$, respectively. ${ }^{6}$ Considering the $\mathrm{CEY}$ measurements from a $\mathrm{Ni}$ foil in the He environment, one can expect the gas amplification of approximately 155 with $\mathrm{Ni}$ KLL Auger electrons $\left(E_{\mathrm{k}}=\right.$ $6400 \mathrm{eV}$ ), while the gain is unity for the electrons that have kinetic energies that are insufficient for the ionization of He.

The averaged attenuation length with the CEY method can be expressed by the following equation; a greater attenuation length 
can be expected compared to that obtained with the TEY method, owing to the weighted fraction of energetic electrons due to the large gas amplification factor.

$$
\tau_{\mathrm{CEY}}=\frac{\int G\left(E_{\mathrm{k}}\right) N\left(E_{\mathrm{k}}\right) \lambda\left(E_{\mathrm{k}}\right) \mathrm{d} E_{\mathrm{k}}}{\int G\left(E_{\mathrm{k}}\right) N\left(E_{\mathrm{k}}\right) \mathrm{d} E_{\mathrm{k}}}
$$

The probing depth of $\mathrm{x}$-ray analysis with the EY method is determined both by the penetration depth of incident X-rays $\tau_{\mathrm{x}}$ and by the attenuation length of the emitted electrons $\tau_{\mathrm{e}}$.

$$
\frac{1}{\tau}=\frac{1}{\tau_{\mathrm{x}}}+\frac{1}{\tau_{\mathrm{e}}}
$$

In general $\tau_{\mathrm{e}}$ is much smaller than $\tau_{\mathrm{x}}$, and the surface sensitivity of the measurements is guaranteed by the attenuation length of the electrons. However, in a special case the total reflection of $\mathrm{X}$-rays can be utilized for reducing the practical analyzing depth. The following expression gives EY from a uniform sample of thickness $t$ as a function of glancing angle $\theta$ and the analyzing depth $\tau$.

$$
I_{\mathrm{EY}}(\theta)=k I_{0} T(\theta) \int_{0}^{\mathrm{t}} e^{-z / \tau} \mathrm{d} z
$$

$I_{0}$ is the intensity of the incident $\mathrm{x}$-rays, and $T(\theta)$ is the gain of the beam intensity at the sample surface. The gain of the beam intensity is calculated from the interference of incident and reflected x-ray beams, ${ }^{14}$ and it will have the maximum value at the critical angle of total reflection. The penetration depth of the incident x-rays $\tau_{\mathrm{x}}$ is greatly reduced under the total reflection condition, and it can be calculated from the equations reported previously. ${ }^{14}$

When the x-ray reflectivity is negligibly small, $T(\theta)$ becomes unity and $\tau_{\mathrm{x}}$ becomes $\sin \theta / \mu$ where $\mu$ is the linear absorption coefficient of x-rays. Whenever $\tau_{\mathrm{x}}$ is much greater than $\tau_{\mathrm{e}}$, the Eq. (4) becomes the following simpler form.

$$
I_{\mathrm{EY}}=k I_{0}\left(1-e^{-\mathrm{t} / \tau_{\mathrm{e}}}\right)
$$

\section{Experimental}

\section{Materials}

$\mathrm{Ni}$ films were formed onto the $\mathrm{Si}$ substrates by the vacuum evaporator equipped with the e-gun excitation. $\mathrm{Si}$ substrates were cut from a commercial Si wafer (Shin-Etsu Chemical Co., Ltd.), and the natural oxidation layer of the wafer was not removed before the $\mathrm{Ni}$ evaporation. A commercial $\mathrm{Ni}$ rod (Nilaco) of high purity was used as a target. The film thickness was monitored by the quartz oscillation gauge. A patterned $\mathrm{Ni}$ film was fabricated, that contains strips of different Ni thickness. The same $\mathrm{Si}$ substrate was exposed several times, and the exposed regions were restricted by the $\mathrm{Al}$ masks each time.

\section{Equipment}

CEY was measured by using the attachment shown in Fig. 1a). An Al coated Mylar film was used as an electrode, and the electrode was placed $2 \mathrm{~mm}$ from a $\mathrm{Cu}$ base. The film electrode was supported with a $\mathrm{Cu}$ flame that has a central hole of $40 \mathrm{~mm}$ in diameter, and the film was transparent both to the incident $\mathrm{X}$-rays and the $\mathrm{Ni} \mathrm{K}_{\alpha} \mathrm{X}$-ray fluorescence. The $\mathrm{Cu}$ base was connected to the current amplifier, and the sample was fixed to the $\mathrm{Cu}$ base with silver paste. A bias voltage of $36 \mathrm{~V}$ was applied from arrayed batteries, and the saturation of the CEY was confirmed in advance with the variable DC source.

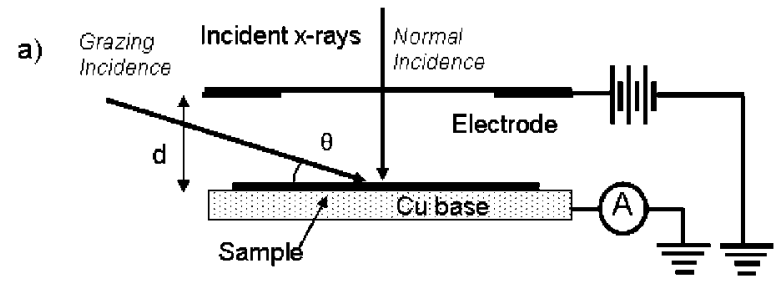

b)

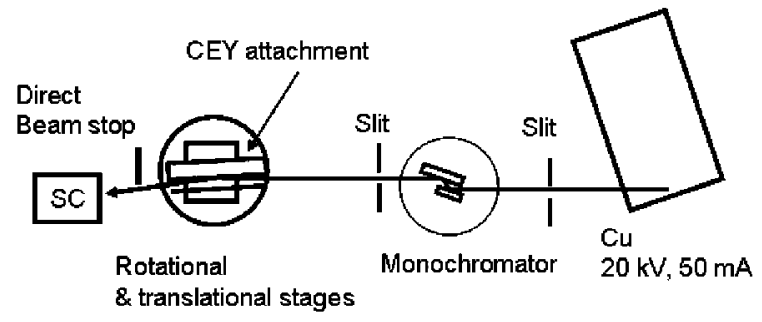

Fig. 1 a) An attachment for conversion electron yield measurements that can be used both in the normal incidence and the grazing incidence conditions. An Al coated Mylar film was placed $2 \mathrm{~mm}$ apart from the $\mathrm{Cu}$ base plate, and a bias voltage of $36 \mathrm{~V}$ was applied. b) A schematic experimental setup for grazing incidence measurements.

\section{XAFS measurements and CEY imaging}

XAFS measurements were carried out on the BL4A of the Photon Factory, Tsukuba, Japan. The storage ring was operated at $2.5 \mathrm{GeV}$, and the synchrotron radiation from a bending magnet was monochromatized with a $\mathrm{Si}(111)$ double-crystal monochromator. Energy of the incident $\mathrm{x}$-rays was calibrated with a transmission spectra of a $\mathrm{Cu}$ foil of $10 \mu \mathrm{m}$ in thickness. The energy of a small peak in the absorption edge of the spectrum was set to be $8.975 \mathrm{keV}$. The beamline's XRF measurement system was used for XRF imaging of the sample. The beam size was defined to be $0.13 \mathrm{~mm}(\mathrm{H})$ and $0.6 \mathrm{~mm}(\mathrm{~V})$ by an XY slit in the experimental hutch. The intensity of the incident $\mathrm{X}$-rays was monitored with an air-filled ionization chamber, and the $\mathrm{x}$-ray fluorescence (XRF) was measured with a $\mathrm{Si}(\mathrm{Li})$ detector.

To realize one dimensional imaging with the CEY, the CEY attachment was placed onto the $\mathrm{X}$ scanning stage, and the CEY from the sample were measured as a function of the sample position. The measurements were carried out under the atmospheric condition.

\section{Total reflection measurements}

Figure 1b) shows a schematic setup of the CEY measurements under the grazing incidence condition. The CEY attachment was placed onto the rotational stage and the translational stage. A conventional sealed $\mathrm{x}$-ray tube ( $\mathrm{Cu}$ line focus) was combined with a Ge(111) channel cut monochromator, and the monochromatized $\mathrm{Cu} \mathrm{K} \mathrm{K}_{\alpha}(8.04 \mathrm{keV})$ and $\mathrm{K}_{\beta}(8.90 \mathrm{keV})$ lines were utilized for the excitation conditions below and above the absorption edge of $\mathrm{Ni}$. The applied bias voltage of the $\mathrm{x}$-ray tube was limited to $20 \mathrm{keV}$ to remove the influence of the third order diffraction at the monochromator. The sample surface was aligned to the rotational center by the translational stage, and the intensity of the reflected beam was monitored with a scintillation counter (SC). 


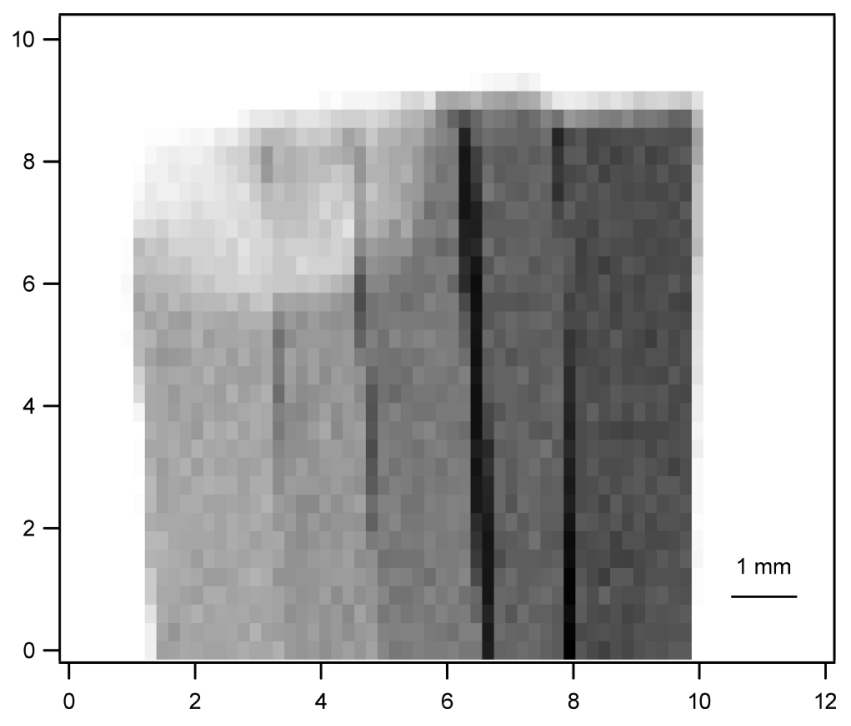

Fig. 2 An X-ray fluorescence image of a patterned $\mathrm{Ni}$ film on the $\mathrm{Si}$ substrate.

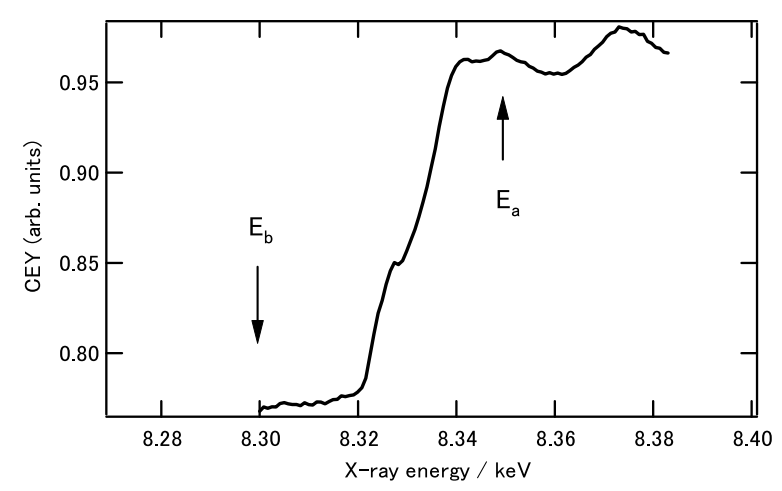

Fig. 3 Ni K edge XAFS spectrum of Ni thin film obtained with the CEY method.

\section{Results and Discussion}

\section{CEY imaging of the patterned Ni film}

Figure 2 shows a Ni $\mathrm{K}_{\alpha} \mathrm{XRF}$ image of the patterned Ni sample obtained with $10 \mathrm{keV} x$-rays. The thickness of the strips was $30,40,50,60$ and $70 \mathrm{~nm}$ from left to the right in the figure. Some of boundary regions were doubly evaporated owing to the misalignment of the mask during the evaporations. Figure 3 shows Ni K edge XAFS spectra from a Ni thin film of $18 \mathrm{~nm}$ measured with the CEY method. Obtained fine structures in the XAFS spectrum are equivalent to those obtained with the conventional transmission method, and the increase of the CEY can be attributed to the creation of KLL Auger electrons and their cascade. Incident x-ray energies of $8.300 \mathrm{keV}\left(E_{\mathrm{b}}\right)$ and $8.350 \mathrm{keV}\left(E_{\mathrm{a}}\right)$ were used for excitation conditions below and above the Ni K edge, and the difference of the obtained data between these two conditions can be attributed to the contribution of Ni KLL Auger electrons.

Figure 4 shows the horizontal line profiles of the patterned sample obtained with the CEY method. The same region was scanned with the X-rays below and above the Ni K absorption edge. The profile of the same region taken previously with the $\mathrm{x}$-ray fluorescence yield (XFY) was imposed in the figure. The

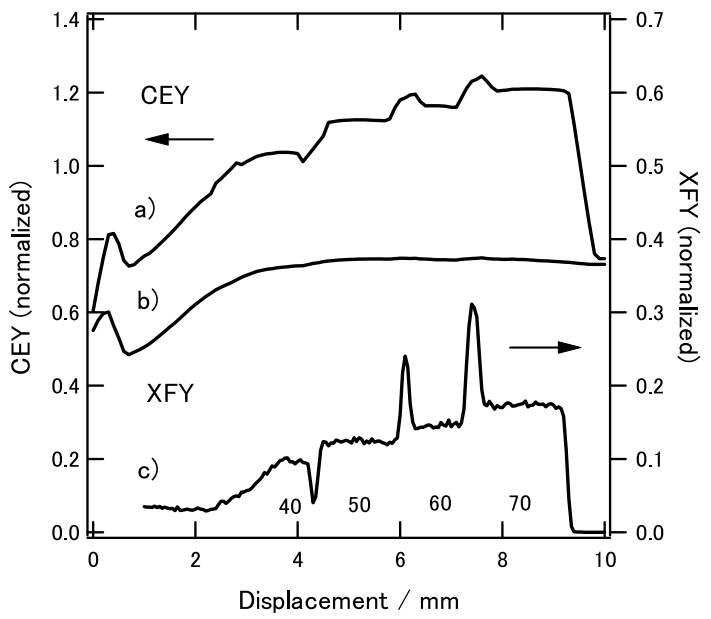

Fig. 4 The line profiles of the CEY from the patterned Ni film obtained with incident $\mathrm{x}$-ray energy a) above and b) below the $\mathrm{Ni} \mathrm{K}$ absorption edge. The line profile shown in c) was obtained with the XRF yield method.

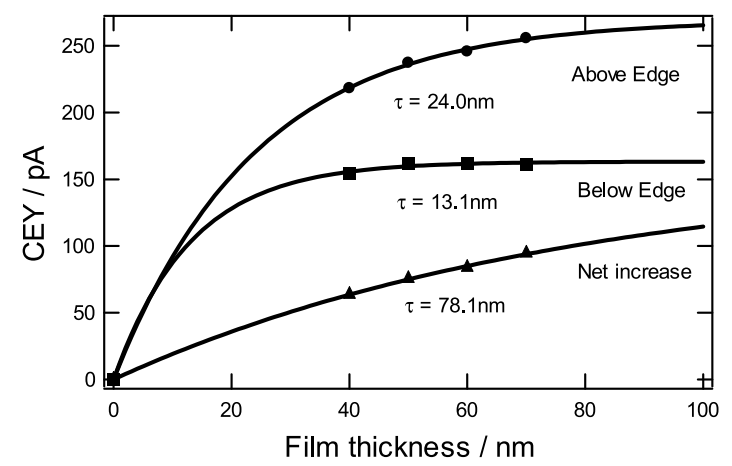

Fig. 5 CEY from a Ni thin film as a function of the film thickness; data obtained with x-rays below (ם), above (๑) the Ni K absorption edge, and net increase attributed to Ni KLL Auger electrons $(\boldsymbol{\bullet})$. Solid lines show interpolation of data obtained with Eq. (5).

region corresponding to the strip of $30 \mathrm{~nm}$ thick was not well evaporated, and the data obtained from strips of 40, 50, 60 and $70 \mathrm{~nm}$ in thickness were used for the further analysis. It was clear that the CEY from the strips of various $\mathrm{Ni}$ thickness showed thickness dependence when the incident $\mathrm{X}$-ray energy above the Ni K absorption edge was employed. However, the CEY was almost constant when the incident $\mathrm{X}$-ray energy was below the absorption edge. The difference of the CEY could be attributed to Ni KLL Auger electrons, since the difference of the beam intensity is negligible between the two energies.

To evaluate the practical analyzing depth with the CEY method, three sets of CEY data were plotted in Fig. 5 as a function of the film thickness of $\mathrm{Ni}$. The third data set was the net increases of the CEY between two conditions. The solid curves represent the results of CEY data fitted to Eq. (5). The evaluated attenuation length $\tau_{\mathrm{e}}$ was $13.1 \mathrm{~nm}$ for the data below the absorption edge, and was $24.0 \mathrm{~nm}$ for the data above the edge. The evaluated attenuation length $\tau_{\mathrm{e}}$ for the net increase was $78.1 \mathrm{~nm}$, and the value is approximately the same as that $(62.4 \mathrm{~nm})$ derived from the empirical equation proposed by Elam et al. ${ }^{7}$ by using kinetic energy of $6340 \mathrm{eV}$ and the density of $8.90 \mathrm{~g} / \mathrm{cm}^{3}$. The contribution of the Ni KLL Auger to the 

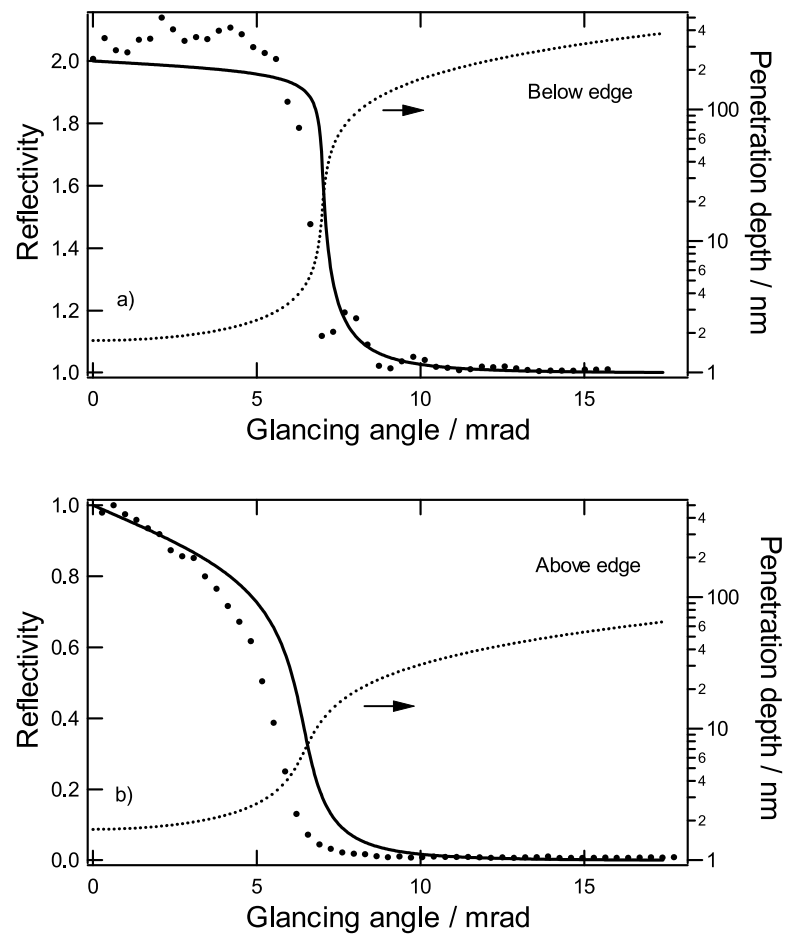

Fig. 6 Calculated (solid line) and observed (dotted line) x-ray reflectivity from a $\mathrm{Ni}$ coated $\mathrm{Si}$ substrate as a function of the glancing angle. The calculated penetration depth of incident x-rays was plotted with the right axis. The complex refractive indices $(n=1-\delta-i \beta)$ used for the calculations were $\delta=2.449310 \times 10^{-5}, \beta=5.147310 \times 10^{-7}$ for $\mathrm{Cu} \mathrm{K}_{\alpha}$ and $\delta=2.07410 \times 10^{-5}, \beta=2.76510 \times 10^{-6}$ for $\mathrm{Cu} \mathrm{K}$.

averaged attenuation length of $24.0 \mathrm{~nm}$ was derived from Eq. (2), and the weighted fraction of NI KLL was estimated to be 0.17 .

\section{CEY under the grazing incidence condition}

As mentioned previously, the analyzing depth of the CEY method can be modified by changing the penetration depth of the incident $\mathrm{x}$-rays. The glancing angle dependence of the CEY from a Ni film was investigated with the experimental setup shown in Fig. 1b). Figure 6 shows observed data of x-ray reflectivity and calculated curves for $\mathrm{x}$-rays below and above the Ni K absorption edge. The glancing angle of the observed data was calibrated with the critical angle of the total reflection where the gain of the beam intensity at the sample surface, $T(\theta)$ has the maximum. The calculations of $x$-ray reflectivity and penetration depth were made for a mirror polished $\mathrm{Ni}$ film of infinite thickness by using equations described previously. ${ }^{14}$ The complex refractive indices $n=1-\delta-i \beta$ for $\mathrm{Cu} \mathrm{K}_{\alpha}(8.04$ $\mathrm{keV})$ and $\mathrm{Cu} \mathrm{K}_{\beta}(8.90 \mathrm{keV})$ were derived from the tabulated complex atomic scattering factors. ${ }^{15}$ Two interference fringes were observed in the reflectivity data from 7 to $10 \mathrm{mrad}$ shown in Fig. 6a), and they were related to the reflection at the interface of $\mathrm{Ni}$ and $\mathrm{Si}$. The calculation (solid curve) was done with the model of infinite thick Ni film, and the effect was not included. The calculated and observed reflectivity shown in Fig. 6b) were smaller than unity with the glancing angles below the critical angle of the total reflection. The reflectivity was affected with the strong absorption of $\mathrm{Ni}$.

Figures 7 and 8 show observed angular dependence of the $\mathrm{CEY}$ as a function of the glancing angle. These profiles showed maximum of the CEY around the critical angle of the total

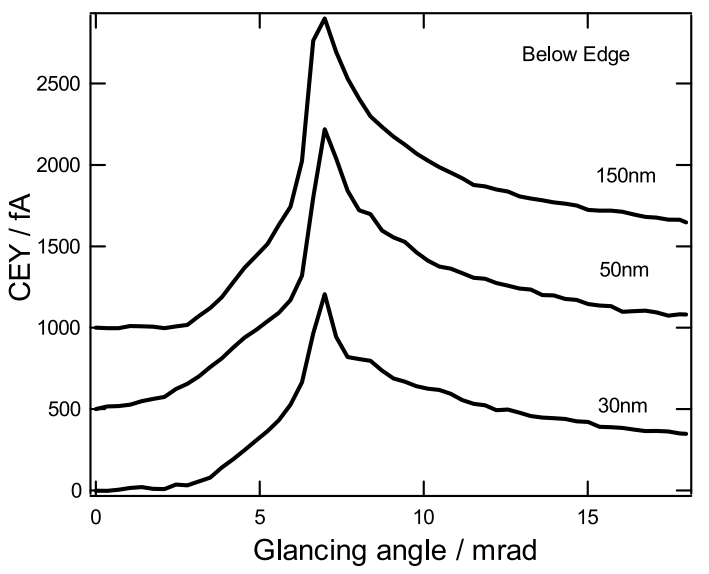

Fig. 7 Glancing angle dependences of CEY from Ni films of 30, 50, $100 \mathrm{~nm}$ on the Si substrates. Incident $\mathrm{X}$-ray energy used was below the Ni K absorption edge (8.04 keV).

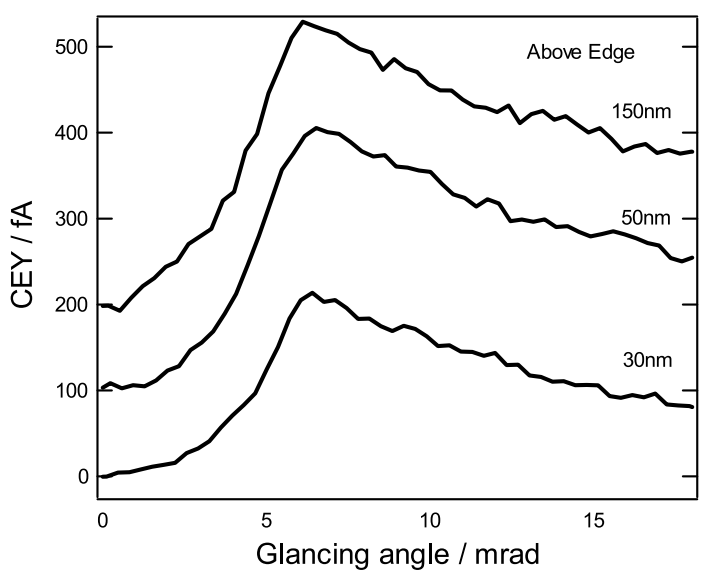

Fig. 8 Glancing angle dependences of CEY from Ni films of 30, 50, $100 \mathrm{~nm}$ on the Si substrates. Incident $\mathrm{X}$-ray energy used was above the Ni K absorption edge (8.91 keV).

reflection, and the results indicated that the electrons were emitted from the near surface regions that could be effectively excited under the total reflection conditions. As can be predicted from the fact that the evaluated attenuation length $\tau_{\mathrm{e}}$ was less than $30 \mathrm{~nm}$, the CEY and the shape of the profiles were almost independent from the thickness of the Ni films.

Figure 9 shows comparisons between the observed data with $30 \mathrm{~nm}$ thick Ni film and the calculated CEY according to the Eq. (4). The $\tau_{\mathrm{e}}$ of 13 and $24 \mathrm{~nm}$ were used for calculations with the conditions below and above the absorption edge. It is clear that the angular dependence of the CEY below the absorption edge is well explained with the calculation. The result suggests that the model was adequate and that $\tau_{\mathrm{e}}$ could be treated as constant during the angular scan. However, some discrepancy was observed for the data with x-rays above the absorption edge. The dotted line shows a calculated curve derived from the weighted sum of the calculated two curves corresponding to $\tau_{\mathrm{e}}=13$ and $78 \mathrm{~nm}$ (Ni KLL Auger). The fractions of the two curves were 0.83 and 0.17 , respectively. The resultant curve was almost the same as that calculated with the single $\tau_{\mathrm{e}}$ of $24 \mathrm{~nm}$. The discrepancy might be attributed to the fact that the fraction of the Ni KLL Auger electrons might be changed as a 

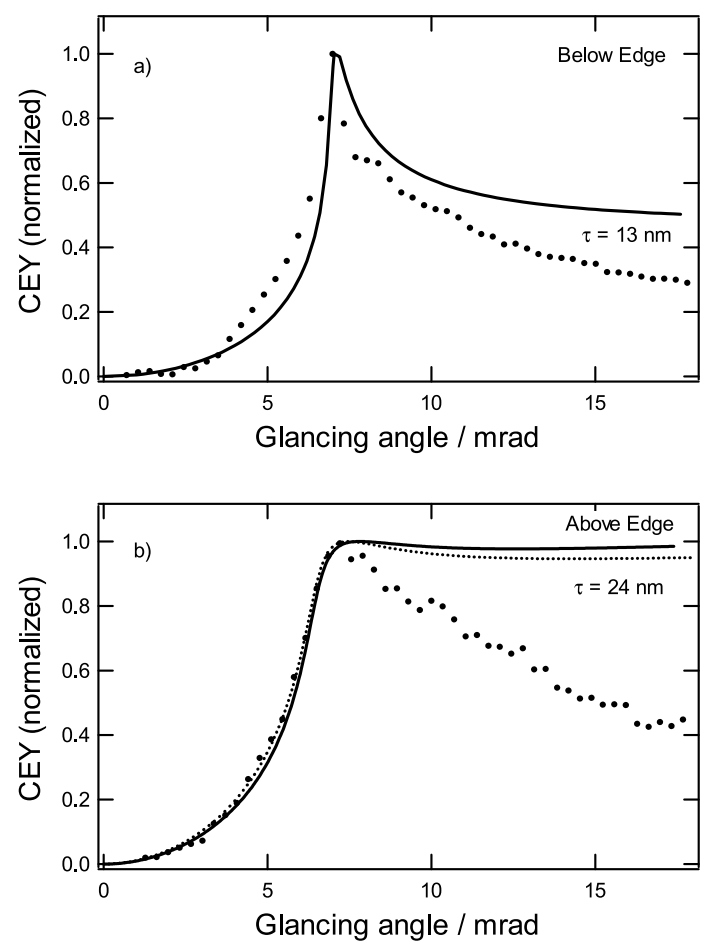

Fig. 9 Comparisons of observed (dots) and calculated (solid lines) glancing angle dependence of the CEY from a Ni film of $30 \mathrm{~nm}$ on the $\mathrm{Si}$ substrate. The incident $\mathrm{x}$-ray energies are a) below $(8.04 \mathrm{keV})$ and b) above $(8.91 \mathrm{keV})$ the $\mathrm{Ni} \mathrm{K}$ absorption edge. The dotted curve in b) was calculated from the weighted sum of two curves corresponding to the Ni KLL Auger and the other electrons.

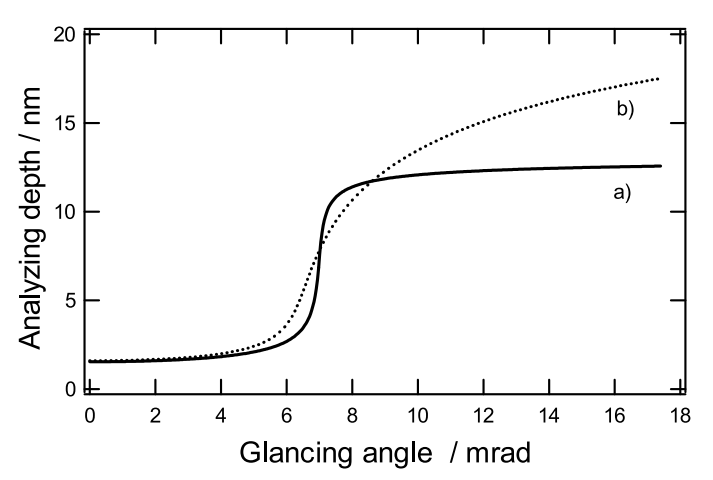

Fig. 10 Evaluated analyzing depth for a Ni thin film with the CEY method around the critical angle of total reflection with the incident $\mathrm{x}$-ray energy above and below the Ni K absorption edge.

function of the glancing angle.

Figure 10 shows the calculated curves for the probing depth $\tau$ according to Eq. (3). $\tau_{\mathrm{e}}$ of 13.1 and $24.0 \mathrm{~nm}$ were used for incident $\mathrm{X}$-rays below and above the $\mathrm{Ni} \mathrm{K}$ absorption edge, and the angular dependent terms $\tau_{\mathrm{x}}$ for both conditions are shown in Fig. 6. The probing depth can be minimized to be around $1.6 \mathrm{~nm}$ under the total reflection condition in both cases. When the X-rays below the Ni K absorption edge are employed, the probing depth can be saturated to be $13 \mathrm{~nm}$ in the higher glancing angle regions. However, the probing depth gradually increases when the $\mathrm{x}$-rays above the $\mathrm{Ni} \mathrm{K}$ absorption edge are employed. It will keep increasing till the probing depth will be determined by the attenuation length of $24 \mathrm{~nm}$.

\section{Conclusions}

The averaged attenuation length with the CEY method was evaluated from the CEY image of a patterned Ni film. The contribution of Ni KLL Auger electrons was investigated by changing the incident $\mathrm{x}$-ray energy around the Ni $\mathrm{K}$ absorption edge. The averaged attenuation lengths increased from 13.1 to $24.0 \mathrm{~nm}$ with the excitation conditions below and above the $\mathrm{Ni} \mathrm{K}$ absorption edge. The increase of the attenuation length was attributed to the large attenuation length $(78.1 \mathrm{~nm})$ of the $\mathrm{Ni}$ KLL Auger electrons. The modification of the analyzing depth with the CEY method was investigated with the grazing incidence condition. The glancing angle dependence of the CEY was compared with the model calculation, and the analyzing depth of around $1.6 \mathrm{~nm}$ was realized under the total reflection condition. The analyzing depth was controlled from $1.6 \mathrm{~nm}$ to the attenuation length by changing the glancing angle, and the possibilities of variable analyzing depth were demonstrated.

\section{Acknowledgements}

Experiments at Photon Factory were carried out under the approval of the Photon Factory Program Advisory Committee (1999G293). We are grateful to Prof. Atsuo Iida at Photon Factory for helping us with the experiments. This work was supported by a Grant-in-Aid for Scientific Research under Grant No. 13650870 from the Ministry of Education, Culture, Sports, Science and Technology (MEXT).

\section{References}

1. J. Stohr, "NEXAFS Spectroscopy", 1992, Chap. 5, Springer-Verlag, Berlin.

2. W. Gudat and C. Kunz, Phys. Rev. Lett., 1972, 29, 169.

3. J. Kawai, S. Hayakawa, Y. Kitajima, S. Suzuki, K. Maeda, T. Urai, H. Adachi, M. Takami, and Y. Gohshi, Proceeding of the Japan Academy, 1993, 69B, 179.

4. J. Kawai, S. Hayakawa, Y. Kitajima, and Y. Gohshi, Anal. Sci., 1995, 11, 519.

5. A. Erbil, G. S. Cargill III, R. Frahm, and R. F. Boehme, Phys. Rev. B, 1988, 37, 2450.

6. G. F. Knoll, "Radiation Detection and Measurement", 2nd ed., 1989, John Wiley \& Sons, Singapore, 132.

7. W. T. Elam, J. P. Kirkland, R. A. Neiser, and P. D. Wolf, Phys. Rev. B, 1988, 38, 26.

8. G. D. Moggridge, S. L. M. Schroeder, R. M. Lambert, and T. Rayment, Nucl. Instrum. Methods B, 1995, 97, 28.

9. S. L. M. Schroeder, G. D. Moggride, R. M. Ormerod, T. Rayment, and R. M. Lambert, Surf. Sci., 1995, 324, L371.

10. S. Hayakawa, J. Noda, and Y. Gohshi, Spectrochim. Acta, Part B, 1999, 54, 235.

11. G. G. Long, D. A. Fischer, J. Kruger, W. T. Elam, and G. A. Prinz, J. Appl. Phys., 1989, 73, 6763.

12. S. Hayakawa, I. Ishii, J. Noda, and Y. Gohshi, Jpn. J. Appl. Phys., 1999, 38, 2161.

13. D. Briggs and M. P. Seah, "Practical Surface Analysis", 2nd ed., 1994, John Wiley \& Sons, England.

14. J. Kawai, M. Takami, M. Fujinami, Y. Hashiguchi, S. Hayakawa, and Y. Gohshi, Spectrochim. Acta, Part B, 1992, 47, 983.

15. S. Sasaki, KEK Report, 1989, 88, 14. 